\title{
A primate restraint and handling system for auditory research ${ }^{1}$
}

DAVID B. MOODY and WILLIAM C. STEBBINS, KRESGE HEARING RESEARCH INSTITUTE, THE UNIVERSITY OF MICHIGAN, Ann Arbor, Michigan 48104, and JOSEF M. M I L L E R , UNIVERSITY OF WASHINGTON, Seattle, Washington, 98105

$A$ set of devices is described that enables daily handling and restraint of large primates. The animals live in cages and wear a special collar that fits into a modified primate restraining chair. A chain and pole arrangement simplifies the daily chairing procedure. A method of head restraint and earphone mounting is also described.

The increasing popularity of large primates as Ss in behavioral research has led to a multitude of different restraint and handling procedures, most of which are based on the use of some form of restraining chair or stand (Findley, 1959; Hardy et al, 1965; Hurst \& Lucero, 1966; Nicholson, 1965; Rahlmann et al, 1964; Thompson et al, 1966; Young, 1957). In many instances, these devices have become permanent housing arrangements for the monkeys, which, with the addition of wheels, are easily portable. Although there is considerable evidence (most of it unpublished) that monkeys in the se devices thrive, grow, and function as well as nonchaired animals, many Es prefer that their animals be more active and not be under continual restraint. One or two procedures have been suggested for the daily chairing of animals that are designed to minimize the risk to the handler (Barrow et al, 1966; Sledjeski, 1969). The advantages of the present handling and restraint system derive from its 7-year developmental history, during which many improvements have been made, and optional special-purpose features not found in previous arrangements have been added.

Our particular application required not only the daily handling of large $(2-9 \mathrm{~kg})$ macaques, but also further restraint of the animal's head so that modified human earphones could be fitted over the external ear to provide a calibrated sound field. The procedures involved are best considered as an integrated system consisting of: (1) a special collar worn by each animal, (2) a chair designed to accept the collar, (3) a technique for chairing the monkey, (4) a method for semiimobilizing the monkey's head, and (5) a mounting arrangement for earphones enabling good acoustic coupling to the monkey's ear.

\section{THE COLLAR}

Each of our monkeys are individually fitted with, and constantly wear, a 4\%-in. square collar made from $1 / 2$-in. thick clear acrylic (Plexiglas). The first step in the construction of these collars is to determine approximate neck measurements and then to cut an elliptical hole in the plastic square. The hole should be large enough to allow the collar to turn freely on the monkey's neck, but not large enough to allow the monkey's lower jaw to fit through. If the head restraint procedure described below is to be used, the hole should be located within $3 / 8$ in. of the front edge of the collar. After the hole is cut, the square is bisected along the minor axis of the ellipse, the comers are rounded, and the edges are fire polished. Two brass plates are fastened to the sides of the collar to join the halves, and 1/8-in. diam brass pins are inserted to strengthen the joint (see inset Fig. 1). Approximately $3 \frac{1}{2} \mathrm{ft}$ of cad nium-plated double loop weldless chain is fastened with a swivel fastener to a brass fitting on the rear edge of the collar. A spring clip fastener is attached to the other end of the chain.

With some practice, our technicians have learned to apply these collars without tranquilizing the monkeys, but when necessary, a $1 \mathrm{mg} / \mathrm{kg}$ i.m. injection of Semylan (phencyclidine hydrochloride, Parke Davis Co.) can be administered. The two halves of the collar are fitted together around the monkey's neck, taking care not to pinch any skin or hair as the halves are joined. The four screws that hold the halves together are then tightened, and the collar is tested for correct fit.

\section{THE CHAIR}

The chair consists of acrylic waist and neck plates and an aluminum seating grid supported on four aluminum uprights (Fig. 1). These uprights are 1/2-in. diam aluminum rods, 36 in. long, which are fastened to a $1 / 4$-in. thick by 19 -in. square aluminum base plate with Flexaframe flange feet (Fisher scientific-Catalog No. 14-666-25). Heavyduty casters are fastened to the underside of the base plate to make the chair easily portable.

The waist and neck plates are constructed from $1 / 2$-in. thick by 15 -in. square acrylic and are fastened to the uprights by means of the Flexaframe flange feet. The distance between the plates is easily adjusted by loosening the setscrews in the flanges and sliding the plates up and down.

A $4 \frac{1}{2} \times 9$ in. slot (A) is cut from the neck plate to accommodate the monkey collar (B). Aluminum strips fastened to the top and bottom of the neck plate along this slot provide the channel into which the collar slides. A $4 \frac{1}{2}$-in. square by $1 / 2$-in. thick acrylic block (C) slides into this slot behind the collar to secure it in place. This block must contain a "U"-shaped notch to provide clearance for the chain fastener on the rear of the collar, as well as mounting bolts for the head holder described below. The block is held in place by an aluminum angle (D) $(3 / 4 \times 1 / 8$ in. thick $\times 10$ in. long), bolted into position with a $1 / 4-20$ thumbscrew at $\mathrm{E}$.

The waist plate contains a 51/4-in. wide by 10 -in. long cutout, which is rounded to a 2 1/8-in. radius. A 7 -in. wide channel is constructed on the top of the waist plate to accommodate the adjustable waist restraint plate (F). This plate is $7 \times 9$ in. and is constructed from $1 / 4$ in. aluminum. It contains a semicircular cutout (2 1/8-in. radius) and a $1 / 4 \times 3$ in. slot through which it is fastened to the chair with a $1 / 4-20$ thumbscrew. The restraint plate may be constructed from $1 / 2$-in. acrylic, but is much stronger if aluminum is used.

The seating grid is constructed from 18-in. long by $1 / 2$-in. diam aluminum rods fastened to the vertical supports with Flexaframe connector blocks (Fisher Catalog No. 14-666-20, or equivalent). We have found it helpful to replace the brass setscrews supplied with the Flexaframe devices with $1 / 4-20$ Allen headset screws. This change requires redrilling and tapping the setscrew holes, but it greatly increases the strength of the system.

\section{HANDLING PROCEDURE}

While the monkeys are in their home cages, the snap fastener at the end of the chain is hooked to the cage mesh, making it impossible for the animal to run freely should the cage door accidentally be opened. This minimizes the risk to people working near the animals, and has had no adverse effects on the animals themselves.

The notable feature of the handling system is a 1 -in. diam tubular steel pole about $2 \frac{1}{2} \mathrm{ft}$ long, one end of which is slotted to fit onto the edge of the monkey collar and the other end of which contains a slot or pin that can be used to lock the chain into position. To remove a monkey 


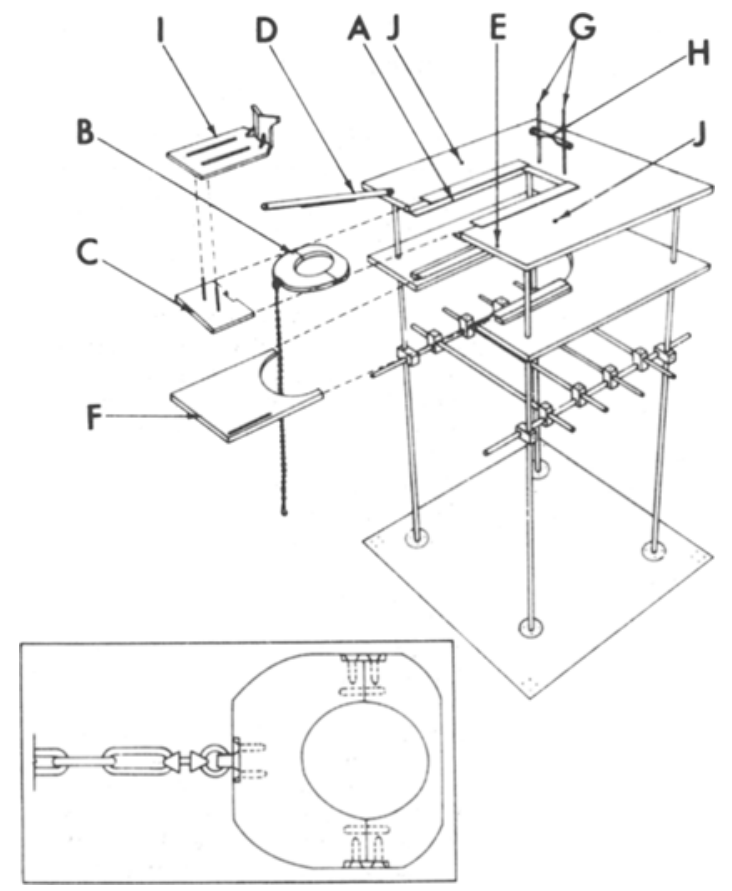

Fig. 1. (Above) The primate restraining chair. Details are included in the text. The inset shows details of the collar worn by each monkey.

Fig. 2. (Right) A monkey in the process of being chaired using the pole.

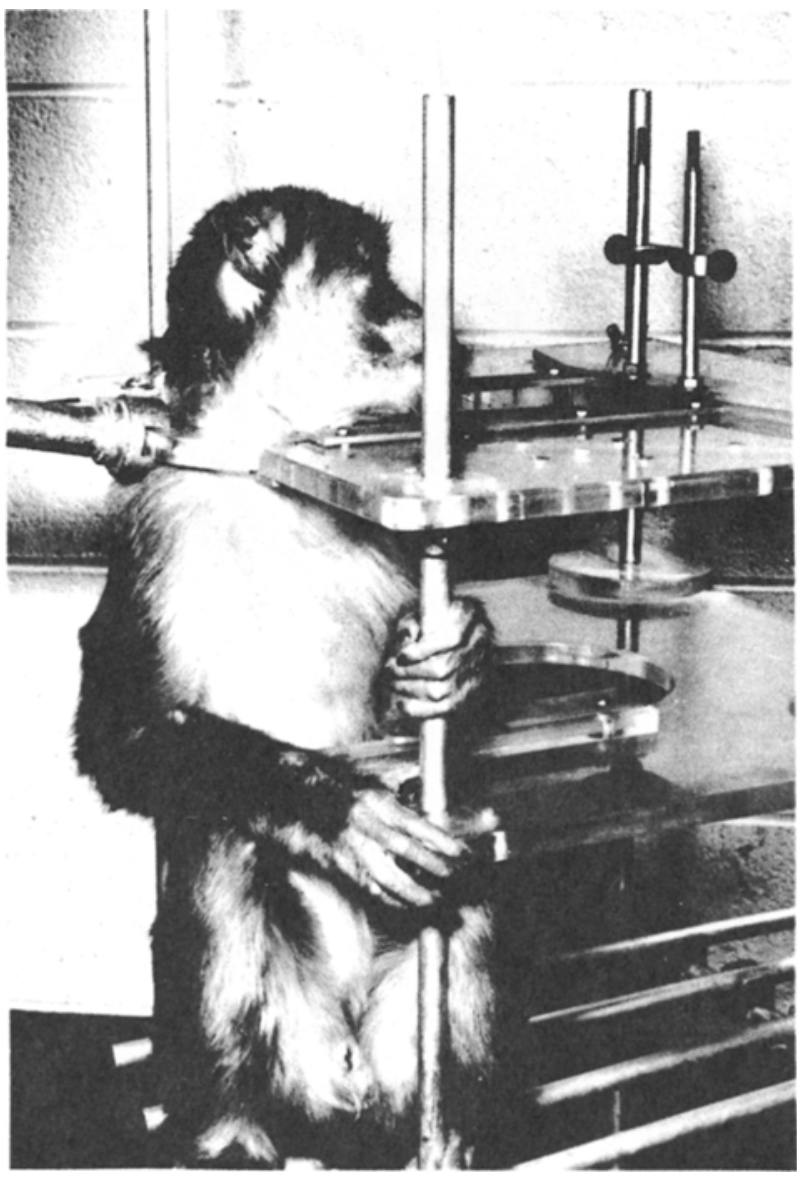

from his cage, the handler simply slides the chain down the center of the pole and locks it into position so that the collar is held rigidly at the end of the pole. If the pole and the chain are of suitable lengths, it is then impossible for the monkey to reach the handler, yet the handler can easily guide the collar into the chair and fasten it in place. Figure 2 shows a monkey in the process of being chaired.

One result of the degree of control that can be exercised over animals using this arrangement is that it soon becomes unnecessary to use the pole. All of our animals now climb out of their cages and into their chairs, where they wait until the handler inserts the collar and fastens it into place.

\section{HEAD RESTRAINT}

In order to mount earphones over the monkey's external ear, it was first necessary to restrict head movements moderately. Head restraint is also useful whenever it is desirable to specify receptor position when using visual stimuli or to minimize movement artifact when recording neuroelectric events. The restraint is accomplished by mounting two $3 / 8$ in diam vertical brass rods $(G)$ in front of the collar slot in the top plate of the chair. The separation of these rods is adjusted to the width of the monkey's muzzle, and a horizontal contoured cross piece $(\mathrm{H})$ is constructed from aluminum to fit over the top of the nose. The cross piece is adjustable up and down to suit individual monkeys. To complete the restraint procedure, a head holder (I) is fastened to the rear of the chair at (C) with wing nuts.

The head holder is also constructed from $1 / 2$-in. acrylic. It consists of a 4 -in. wide $x$ 8 -in. long horizontal base plate and a $2 \frac{1 / 4}{4}$-in. wide by $2 \frac{1}{2}$-in. high " $V$ "-shaped vertical support. The base plate contains two $1 / 4$-in. wide $\times 6$-in. long slots through which it can be fastened to the chair. Viewed from above, the two wings of the vertical support are separated by an angle of about $150 \mathrm{deg}$. This angle may be made more acute and the overall width of the upright increased if headphones are not used.

Care must be taken when placing the monkey in the head holder not to force the animal's head too tightly against the nose pieces. If head restraint is too severe, the animal will be unable to eat, and pressure sores may result.

If the monkey is to be used on a food or liquid reinforcement procedure, the reinforcer must be delivered directly to the animal's mouth. If standard food pellets are used, the delivery chute can be located directly in front of the $S$, who will quickly learn to pick up pellets with his tongue.

\section{EARPHONE MOUNTING}

Since the monkey's head is somewhat free to move, earphones must be mounted so that they are free to follow any motion. Initial attempts to get the monkey to wear headband-mounted human phones were unsuccessful because of the weight. A mounting arrangement such as that shown in Fig. 3 was devised in which a human receiver (Permoflux-PDR-600) is mounted in a yolk arrangement that is adjustable in three dimensions and mounted on a universal swivel joint. This permits enough movement to compensate for reasonable variations in head position. The described mounting is fastened onto the top plate of the chair at (J) with thumbscrews. The phones are fitted firmly over the monkey's ear to provide good acoustic coupling and to further restrict head movement.

Construction details of the headphone mount are shown to scale in Fig. 4. The size of the headphone mounting yolk and 

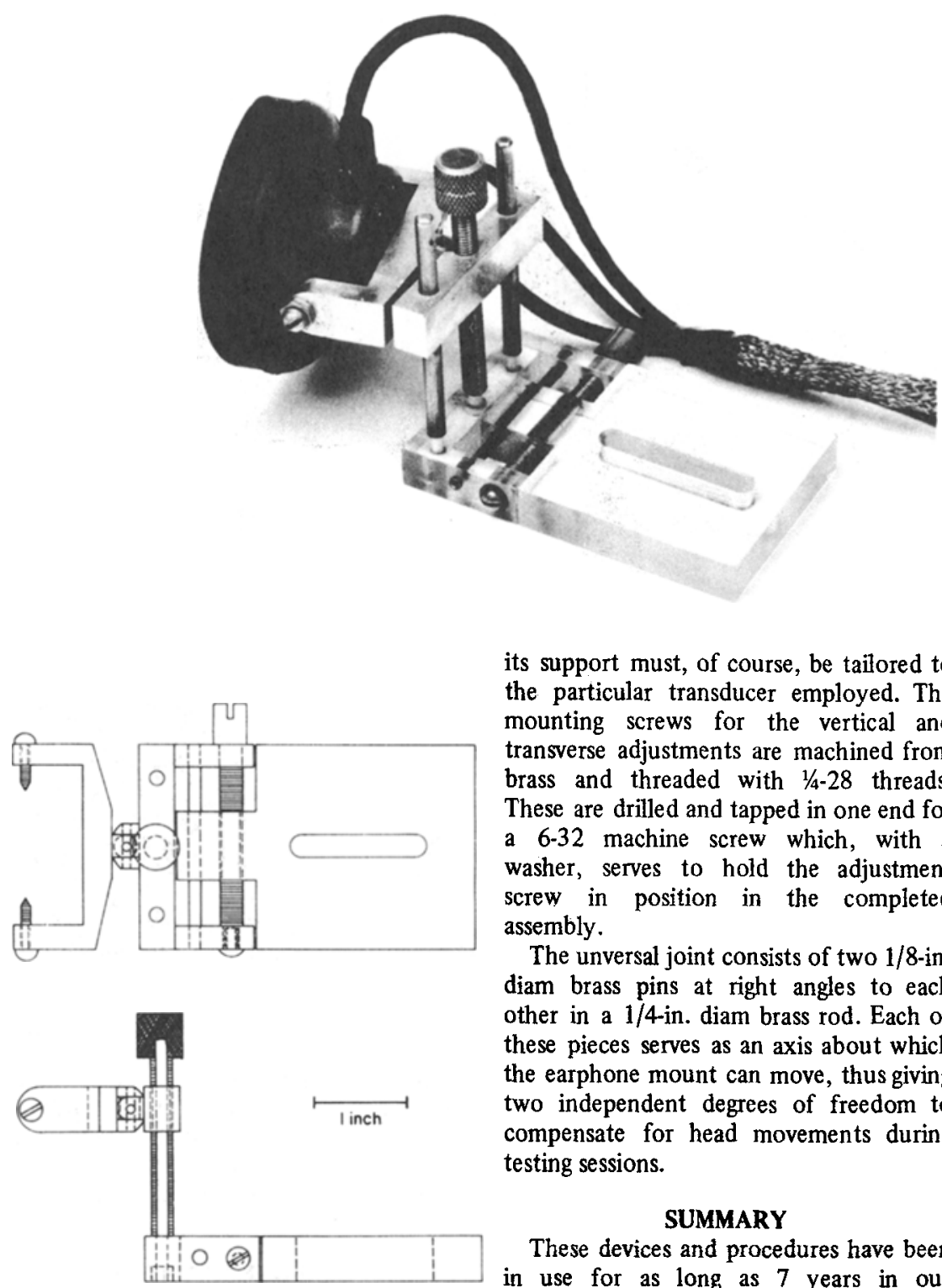

Fig. 4. Scale drawing of the earphone mounting system. Details are included in the text.
Fig. 3. A close-up of the earphone mounting system.

readily adapt to the chairing procedures and to the head restraint, and work for sessions lasting up to $2 \frac{1}{2} \mathrm{~h}$. Deprivation is also easier to control when the animals live in cages instead of chairs.

\section{REFERENCES}

BARROW, S., LUSCHEI, E., NATHAN, M., \& SASLOW, C. A training technique for the daily chairing of monkeys. Journal of the Experimental Analysis of Behavior, 1966, 9, 680.

FINDLEY, J. An intermediate restraining device for working with monkeys. Journal of the Experimental A nalysis of Behavior, 1959, 1, 121 125.

HARDY, J. R., HIROTA, K., HARKINS, H. N. \& NYHUS, L. M. A new chair for the study of restrained monkeys. Medicina et Pharmacologia Experimentalis, 1965, 12, 336-344.

HURST, C. M., \& LUCERO, J. F. A collar and chain arrangement for handling and seating macaca mulatta. Technical Report ARL-TR-66 14. 6571 st Aeromedical Research Laboratory, Hollomon Air Force, New Mexico, 1966.

the particular transducer employed. The mounting screws for the vertical and transverse adjustments are machined from brass and threaded with $1 / 4-28$ threads. These are drilled and tapped in one end for a 6-32 machine screw which, with a washer, serves to hold the adjustment screw in position in the completed assembly.

The unversal joint consists of two 1/8-in. diam brass pins at right angles to each other in a 1/4-in. diam brass rod. Each of these pieces serves as an axis about which the earphone mount can move, thus giving two independent degrees of freedom to compensate for head movements during testing sessions.

\section{SUMMARY}

These devices and procedures have been in use for as long as 7 years in our laboratory with excellent results. They have proven to be simple and safe methods which enable $45-\mathrm{kg}$ female lab technicians to handle large $9-\mathrm{kg}$ macaques without harmful effects on either. The animals
NICHOLSON, A. N. A restraining chair for long-term experimental studies in conscious monkeys. Medical Electronics \& Biological Engineering, 1965, 3, 77-80.

RAHLMANN, D. F., HANSEN, J. T., PACE, N BARNSTEIN, N. J., \& CANNON, M. D. Handling procedures and equipment for physiological studies on the pig-tailed monkey (Macaca nemestrina). Laboratory Animal Care, 1964, 14. $125-130$

SLEDJESKI, M. A monkey chair for temporary restraint with minimal human contact Physiology \& Behavior, 1969, 4, 273-276.

THOMPSON, T., SEAL, D., \& BLOOM, W. A suspended platform for use in chronic restraint of monkeys. Journal of the Experimental Analy sis of Behavior, 1966, 9, 146.

YOUNG, F, A. A primate control system. Proceedings of the Animal Care Panel, 1957, 2 , 127-137.

\section{NOTE}

1. Credit for many of the ideas in design and development of the chair, collar, and pole, and their excellent construction must go to Mr. Franz Weiser, and Mr. Guenther Pickutt in the machine shop of the Kresge Hearing Research Institute. The research and development was supported by NIH Grants NS 05077 and NS 05785. 